\title{
Outcome and predictors of transarterial chemo embolization in decompensate cirrhotic hepatocellular carcinoma patients
}

\begin{abstract}
Background: Transarterial chemo embolization (TACE) is recommended for hepatocellular carcinoma (HCC) patients with well-preserved liver function. In Thailand, TACE is occasionally performed in decompensated cirrhosis due to limited resources. We compared overall survival by TACE in HCC patients with decompensated cirrhosis to those with compensated cirrhosis.

Methods: A retrospective cohort study was conducted at a tertiary hospital. HCC patients were recruited from January 1, 2007 to December 31, 2016 and divided into 3 groups by Child-Pugh scores A, B (7), and B ( $\geq 8)$. Overall survival was the primary outcome. $\mathrm{P}<.05$ was considered statistically significant.

Results: A total of 331 hepatocellular patients were included: Child A (66\%), Child B (7) $(20 \%)$, and Child B ( $\geq 8)(14 \%)$. The mean (SD) follow-up time was 20(16) months and the median survival times were 22,13 , and 12 months in Child A, Child B (7), and B $(\geq 8)$, respectively $(\mathrm{P}<.001)$. In comparison between Child B (7) and Child $\mathrm{B}(\geq 8)$, the overall survival rates did not reach statistical difference $(\mathrm{P}=.91)$. The overall survival rate in a subgroup of patients with Child B (9) was the worst. Predictors for survival were high serum albumin, tumor size $<5 \mathrm{~cm}$, TACE frequency $>2$, multimodality treatment, and absence of complications.

Conclusions: TACE can be performed in HCC patients with early de compensated cirrhosis (Child-Pugh score 7-8) resulting in a 1-year survival close to that of Child A. Regarding conflicting Child-Pugh score recommendation for TACE, our study suggests that TACE can be performed in patients with Child score of up to B8.
\end{abstract}

Keywords: carcinoma, hepatocellular, liver neoplasm, chemo embolization, therapeutic, liver cirrhosis, survival analysis
Volume 9 Issue 6 - 2018

\author{
Natcha Cheewasereechon, Sawangpong \\ Jandee, Teerha Piratvisuth \\ Department of Internal Medicine, Prince of Songkla University, \\ Thailand
}

Correspondence: Natcha Cheewasereechon, NKC Institute of Gastroenterology and Hepatology, Department of Internal Medicine, Prince of Songkla University, Karnjanavanit Rd, Hat Yai, Songkhla, Thailand 901 10 Tel +6674451965, Email natchichee@hotmail.com

Received: June 01, 2018 | Published: November 20, 2018
Abbreviations: TACE, transarterial chemo embolization; HCC, hepatocellular carcinoma

Lay summary: Early de compensated cirrhotic patients with liver cancer have more option of treatment by TACE.

\section{Introduction}

Liver cancer is the sixth most common cancer worldwide and the second most common cause of cancer-related death. More than $90 \%$ of liver cancers are hepatocellular carcinoma $(\mathrm{HCC}) .{ }^{1}$ Staging is currently based on the Barcelona Clinic Liver Cancer (BCLC) staging system which includes tumor burden, liver function, and patient performance status. Treatments are stratified according to the BCLC stages. ${ }^{1,2}$ Tran arterial chemo embolization (TACE) is a modality of choice for HCC patients with BCLC stage B (intermediate stage). TACE can also be applied in very early and early stage $\mathrm{HCC}$ when surgery or locoregional therapies are not eligible due to tumor location or medical comorbidities. ${ }^{3}$ TACE was initially proved to improve survival in HCC by several studies; however, most of the patients in these studies were in Child A. ${ }^{4-7}$ According to the guidelines, TACE is recommended for Child A and highly selected Child B by the American Association for the Study of Liver Disease, ${ }^{1}$ for Child A by the European Association for the Study of the Liver, ${ }^{2}$ and Asian Pacific Association for the Study of the Liver. ${ }^{3}$ Thailand guideline 2015 states that patients with Child $\geq 9$ are an absolute contraindication for TACE.

In Thailand, most HCC patients often come with late presentation; therefore, they tend to be in the intermediate to advanced stage and have impaired liver function. ${ }^{8-12}$ From the experience in our institution, TACE is sometimes applied to patients beyond the criteria due to ineligibility of other loco-regional therapies and limited access to sorafenib or liver transplantation. Some Child-Pugh B patients with scores of 8 or 9 undergo TACE with questionable outcomes. Few studies, so far, have clarified the effect of high Child scores on the outcome of TACE and the results are conflicting. Therefore, we aimed to study the outcomes of TACE in HCC patients in terms of overall survival among different Child scores and to determine the predictors for survival at a tertiary care hospital.

\section{Materials and methods}

A retrospective cohort study was conducted at Songklanagarind Hospital, Prince of Songkla University, Thailand. We recruited patients diagnosed with HCC (ICD10 code C22.0) from January 1, 2007 to December 31, 2016 from the hospital database. The inclusion criteria were patients aged $\geq 18$, first diagnosed with HCC, underwent TACE as monotherapy or combination therapy, and had at least $1 \mathrm{OPD}$ 
visit or at least 1 follow-up imaging after TACE or both. Exclusion criteria were HCC with other active malignancies. Diagnosis of HCC was based on the AASLD guideline. ${ }^{1}$

Patient characteristics, laboratory results, and radiological data were reviewed from the computer-based medical records. Patients were divided into 3 groups by their liver reserve using the ChildPugh scoring system: A, B (7), and B $(\geq 8)$. Comorbidities were cardiovascular, cerebrovascular, and metabolic diseases. Doxorubicin was used as a chemotherapeutic agent in all TACE patients. Patient status at the end of the study (November 30, 2017) was defined as alive or death using data from the Thailand civil registration database. Complications were defined as TACE-related complications or liver decompensating (increase in Child score $\geq 1$ ) at 4-8weeks after TACE. Overall survival was measured from the date of diagnosis to the date of study termination. The study was approved by the Human Research Ethics Committee of Prince of Songkla University.

\section{Statistical analysis}

Statistical analyses were performed using $\mathrm{R}$ program version 3.4.1. Data were demonstrated as mean (SD), median (IQR) or percentage as appropriate. Comparisons of means, medians, and percentages among the 3 groups were done by one-way analysis of variance
(ANOVA), Kruskal-Wallis test and Chi-square test or Fischer's exact test, respectively. Overall survival was analysed by the Kaplan Meier model and compared by log rank test. Predictors for survival were explored using Cox's proportional hazard model. $\mathrm{P}<.05$ was considered statistically significant.

\section{Results}

After searching the hospital database and performing a medical record review, 331 hepatocellular patients who underwent TACE were included in the study. The mean (SD) age was 60(11). Most of the patients were male (72\%) and $90 \%$ of the patients had cirrhosis. About half of the patients had hepatitis B (49\%) as a HCC risk factor, followed by alcohol (23\%), hepatitis C (20\%), and nonalcoholic fatty liver disease $(8 \%)$. When the patients were grouped according to their liver function reserves by the Child-Pugh score, the percentages of patients who were Child A, Child B (7), and Child B $(\geq 8)$ were $66 \%, 20 \%$, and $14 \%$, respectively. The majority of the patients in each group were BCLC stage B. The percentage of patients who had TACE as monotherapy was $64 \%$, while the others had multimodality therapy including liver resection, percutaneous ethanol injection, radiofrequency ablation, and sorafenib. The mean TACE frequency was 2. The mean (SD) follow-up time was 20(16) months. Patient baseline characteristics are shown in Table 1.

Table I Baseline characteristics of patient population

\begin{tabular}{|c|c|c|c|c|}
\hline & Child A & Child B(7) & Child B( $\geq 8)$ & \\
\hline & $n=219(66 \%)$ & $n=67(20 \%)$ & $n=45(14 \%)$ & $r$ value \\
\hline Male gender, $\mathrm{n}(\%)$ & $162(74)$ & $46(69)$ & $29(64)$ & 0.36 \\
\hline Age $(\text { year })^{* * *}$ & $59(11)$ & $63(10)$ & $60(10)$ & 0.09 \\
\hline \multicolumn{5}{|l|}{ Risk factors } \\
\hline Hepatitis B, n(\%) & $116(53)$ & $28(42)$ & $18(40)$ & 0.12 \\
\hline Hepatitis C, n(\%) & $36(16)$ & $19(28)$ & $12(27)$ & 0.05 \\
\hline NAFLD, n(\%) & $15(7)$ & $7(10)$ & $3(7)$ & 0.6 \\
\hline Alcohol, n(\%) & $56(26)$ & $15(22)$ & $6(13)$ & 0.21 \\
\hline Cirrhosis, n(\%) & $189(87)$ & $66(99)$ & $44(98)$ & $.003 *$ \\
\hline Ascites, $\mathrm{n}(\%)$ & $26(12)$ & $55(82)$ & 40(89) & $<.00 I^{*}$ \\
\hline Comorbidities, n(\%) & $66(30)$ & $23(34)$ & $18(40)$ & 0.4 \\
\hline \multicolumn{5}{|l|}{ Lab results } \\
\hline $\mathrm{AST}(\mathrm{U} / \mathrm{L}) * *$ & $57(39,94)$ & $69(50,103)$ & $87(54,152)$ & $<.001 *$ \\
\hline $\operatorname{ALT}(\mathrm{U} / \mathrm{L})^{* *}$ & $39(26,59)$ & $37(24,52)$ & $39(29,68)$ & 0.63 \\
\hline $\operatorname{ALP}(\mathrm{U} / \mathrm{L})^{* *}$ & $122(93,187)$ & $150(101,199)$ & $169(130,226)$ & $.001 *$ \\
\hline Total bilirubin(mg/dL)** & $0.7(0.5, I .1)$ & $1.0(0.8,1.5)$ & $2.3(1.4,2.8)$ & $<.00 I^{*}$ \\
\hline Albumin $(\mathrm{g} / \mathrm{dL})^{* *}$ & $3.7(3.5,4.1)$ & $3.1(2.9,3.3)$ & $2.7(2.5,3.2)$ & $<.001 *$ \\
\hline INR** & I.I(I.I,I.2) & $1.3(1.2,1.3)$ & $1.3(1.2,1.4)$ & $<.001 *$ \\
\hline Platelet $(* 103 / \mu \mathrm{L}) * *$ & $149(94,227)$ & $1 \mid 5(7|| 66)$, & $88(66, \mid 48)$ & $<.001 *$ \\
\hline $\operatorname{AFP}(n g / m L) * *$ & $68(9, \mid 131)$ & $84(20,1218)$ & $129(\mid 2,2829)$ & 0.49 \\
\hline Tumor number** & $\mathrm{I}(\mathrm{I}, 2)$ & $I(I, 2)$ & $I(I, 3)$ & 0.13 \\
\hline Tumor size $(\mathrm{cm})^{* *}$ & $4.3(2.5,8.8)$ & $4.8(3,8.3)$ & $4.9(2.4,6)$ & 0.62 \\
\hline Multifocal tumor, n(\%) & $99(45)$ & $38(57)$ & $26(58)$ & 0.13 \\
\hline
\end{tabular}


Table Continued...

\begin{tabular}{|c|c|c|c|c|}
\hline & Child A & Child B(7) & Child $B(\geq 8)$ & $P$ value \\
\hline Portal vein thrombosis, $\mathrm{n}(\%)$ & $49(22)$ & $14(2 \mid)$ & II(24) & 0.91 \\
\hline Main portal vein, $\mathrm{n}(\%)$ & $7(14)$ & $I(7)$ & $4(36)$ & 0.14 \\
\hline Portal vein branch, $\mathrm{n}(\%)$ & $47(96)$ & $14(100)$ & $10(91)$ & 0.46 \\
\hline \multicolumn{5}{|l|}{ Stage } \\
\hline BCLC Stage 0, n(\%) & $18(8)$ & $0(0)$ & $0(0)$ & $.004 *$ \\
\hline BCLC Stage A, n(\%) & $67(31)$ & $14(2 \mid .5)$ & $14(3 \mid)$ & 0.35 \\
\hline BCLC Stage B, n(\%) & $87(40)$ & $37(57)$ & $20(44)$ & 0.05 \\
\hline BCLC Stage C, n(\%) & $47(21)$ & $\mid 4(2 \mid .5)$ & II (24) & 0.91 \\
\hline
\end{tabular}

*Statistical significance, **Median (IQR), ***Mean(SD), Multifocal tumor; tumor number $\geq 2$, tumor size; size of the largest tumour.

AST, aspartate transaminase; ALT, alanine transaminase; ALP, alkaline phosphatase; INR, international normalized ratio; AFT, alpha-fetoprotein; BCLC, barcelona clinic liver cancer

For all 331 patients, the median survival time was 17 months. Oneand 2 year survival rates were $59 \%$ and $41 \%$, respectively. In terms of primary outcome, the median survival times were 22 months in Child A, 13months in Child B (7), and 12months in Child B $(\geq 8)$. The 1and 2-year survival rates for Child A, Child B (7), and Child B $(\geq 8)$ were 63 and $48 \%, 51$ and $29 \%$, and 49 and $26 \%$, respectively. Overall survival was statistically different among the 3 groups by log rank test $(\mathrm{P}<.001)$ as shown in Figure 1. In comparison between the Child B (7) and Child $\mathrm{B}(\geq 8)$ groups, the overall survival rates did not reach statistical difference by log rank test $(\mathrm{P}=.91)$.

When we re-stratified the patients into Child A, Child B (7-8) and Child B (9), a statistical difference in overall survival was found with the worst survival in Child B (9) $(\mathrm{P}<.001)$ as shown in Figure 2. The median survival time was 22 months in Child A, 13months in Child

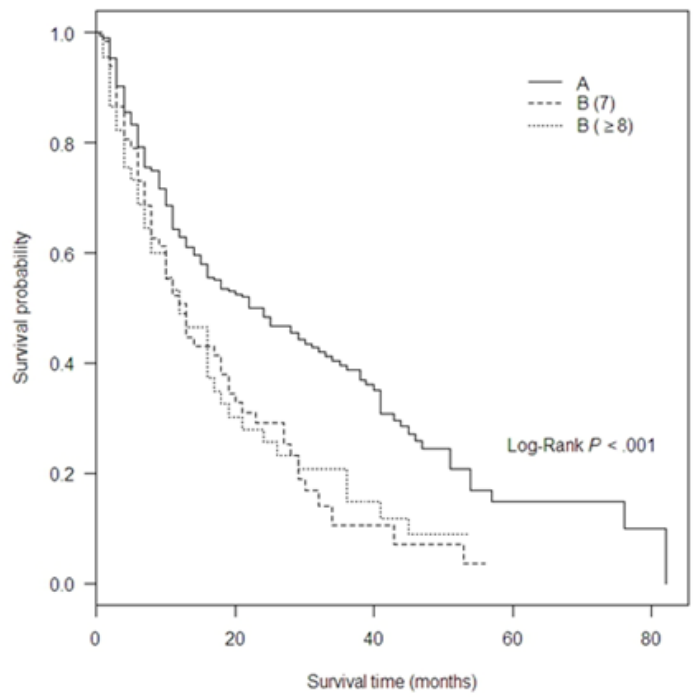

Figure I Kalpan Meier curves of overall survivals in child A,B (7), B( $\geq 8)$.
B (7-8), and 8months in Child B (9). The 1 and 2year survival rates for Child B (7-8) were 52 and $30 \%$, respectively, while the 1-year survival rate for Child (9) was $27 \%$. In a comparison between Child B (7-8) and Child B (9), overall survival did not reach statistical difference by $\log$ rank test $(\mathrm{P}=.14)$.

TACE frequency, multimodality treatment, and complications, including liver de compensation, were not different among the 3 groups. Death outcome was more common in Child B ( $\geq 8)$ and B (7) than in Child (A). Most of the patients in each group had progressive disease according to the modified Response Evaluation Criteria in Solid Tumors (Table 2). By multivariate analysis, high serum albumin, tumor size $<5 \mathrm{~cm}$, TACE frequency $>2$, multimodality treatment and absence of complications were predictors for survival (Table 3 ).

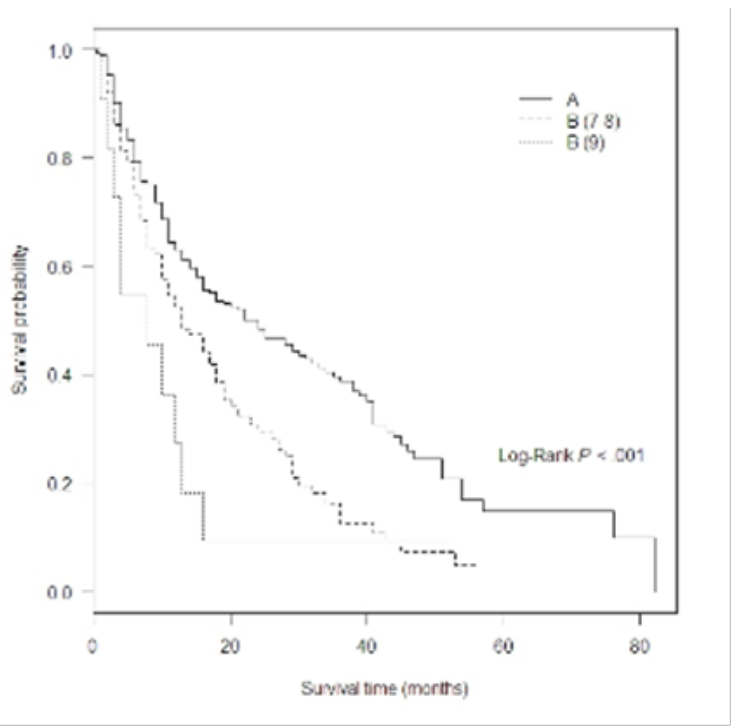

Figure 2 Kalpan Meier curves of overall survivals in child A, B (7-8), B(9). 
Table 2 Treatment and outcomes

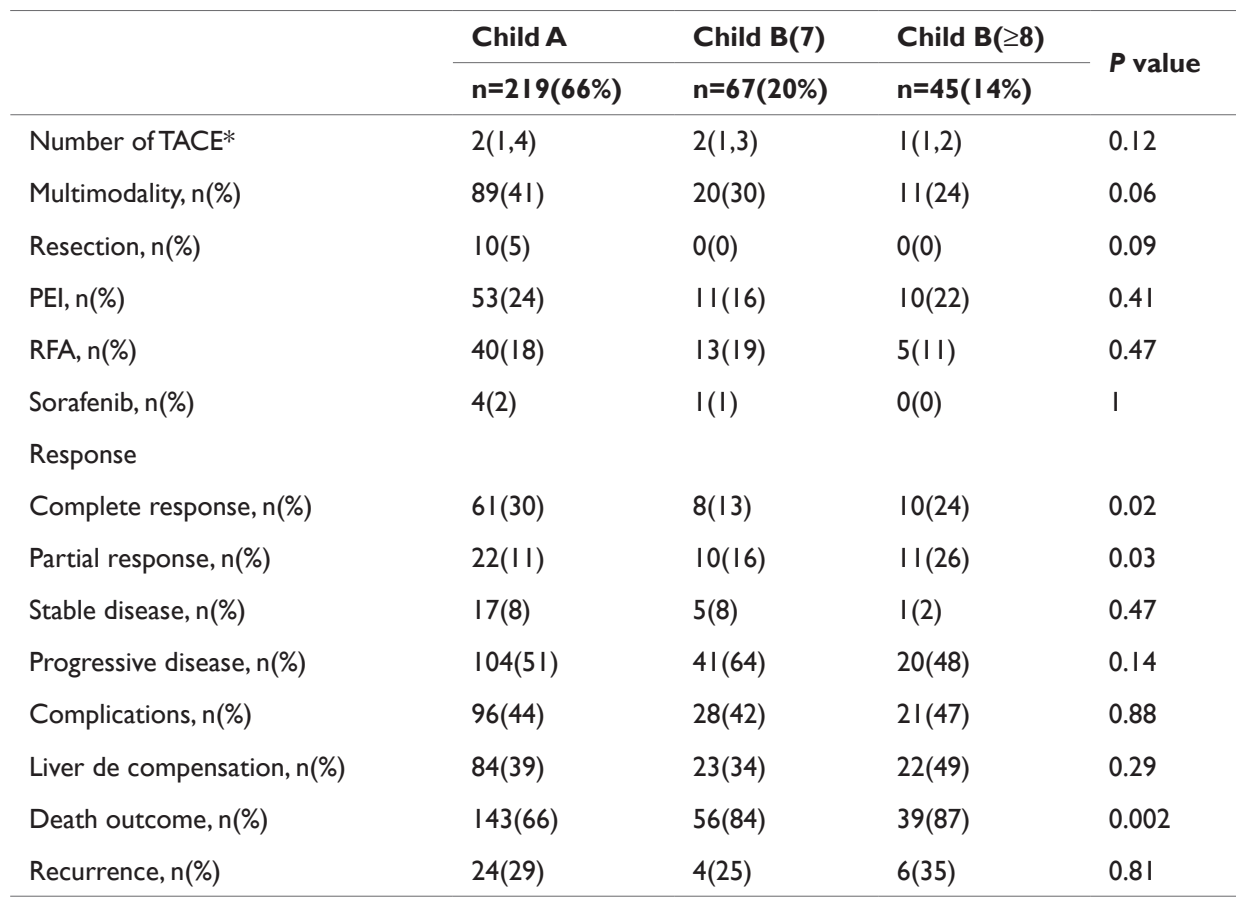

*Median (IQR)

TACE, transarterial chemoembolization; PEI, percutaneous ethanol injection; RFA, radiofrequency ablation

Table 3 Predictors for survival

\begin{tabular}{lll}
\hline Multivariate & HR(95\% CI) & P value \\
\hline Albumin & $0.52(0.4 I, 0.65)$ & $<.00 \mathrm{I}$ \\
Tumor size $>5 \mathrm{~cm}$ & $2.18(\mathrm{I} .63,2.9 \mathrm{I})$ & $<.00 \mathrm{I}$ \\
TACE No. $>2$ & $0.48(0.36,0.63)$ & $<.00 \mathrm{I}$ \\
Multimodality Treatment & $0.4 \mathrm{I}(0.3,0.57)$ & $<.00 \mathrm{I}$ \\
Complications & $\mathrm{I} .67(1.27,2.19)$ & $<.00 \mathrm{I}$
\end{tabular}

\section{Discussion}

TACE has long been a treatment modality for unrespectable HCC Since liver de compensation is one of the complications of concern after TACE, liver function has an impact on the outcome of TACE. De compensated cirrhosis is generally contraindicated in TACE. ${ }^{13}$ Guidelines have suggested that patients with a Child-Pugh score A are good candidates for TACE although some guidelines, such as AASLD, extend the feasibility of TACE to highly-selected Child B patients. Bolondi et al., ${ }^{14}$ proposed a BCLC B sub classification into B1-B4 according to the Child-Pugh score, tumor burden, Eastern Cooperative Oncology Group performance status, and portal vein thrombosis, and recommended TACE for patients up to Child score 7. ${ }^{14}$

To date, few studies have clarified the effect of a high Child score on the outcome of TACE and the results are conflicting. A study by Nishita et al failed to show differences in 30-day mortality and 6-week morbidity between low $(<9)$ and high $(\geq 9)$ Child scores. ${ }^{15}$ A study by Piscaglia et al., ${ }^{16}$ showed that Child scores of B (7) and B (8) tended to have different survival rates but did not reach statistical significance. ${ }^{16}$ Another study by Yamakado et al., ${ }^{17}$ found the Child score to be a prognostic factor in HCC patients undergoing TACE; however, subgroups of Child B $\left(7,8\right.$, and 9 ) were not explored. ${ }^{17}$

From the baseline characteristics of our patients, most of the liver biochemical profiles, the presence of as cites, and cirrhosis were statistically different among the 3 groups, which was compatible with the grading of the Child scores. However, tumor burdens, including size, number, multimodality, and vascular invasion which are important factors affecting survival, did not differ among the 3 groups.

TACE was performed most frequently in patients with BCLC stage $\mathrm{B}(44 \%)$ followed by stage $\mathrm{A}(29 \%)$ which was consistent with the treatment allocation suggested in the BCLC guideline. The median survival time of all patients was 17 months. Since the majority of the patients were BCLC stage B, it corresponded to 16-20months of BCLC stage B reported in the guideline. ${ }^{2}$ Some of the patients with BCLC stage C $(22 \%)$ underwent TACE because of limited access to sorafenib and the results showed a median survival time of 7.5 months, which was comparable to 6.5 months in a study by Cheng et al., ${ }^{18}$ despite fewer Child $\mathrm{A}(65 \%)$ in this study group compared to $97 \%$ of Child A in the study by Cheng et al. This implicated the rational application of TACE in selected patients with BCLC stage C.

The study's primary outcome was overall survival in each group. As expected, Child A group, which was the only group to be proved suitable and recommended for TACE, had the best survival. According to the survival curve that compares Child $\mathrm{A}, \mathrm{B}(7)$, and $\mathrm{B}(\geq 8)$, the log rank test showed statistical difference among the 3 
groups. Surprisingly, the survival curve of B (7) and B ( $\geq 8)$ are almost the same. After we explored the log rank test between B (7) and B $(\geq 8)$, survival did not differ.

In the subgroup analysis of Child score 9, we compared Child $\mathrm{B}(9)$ with Child $\mathrm{B}(7-8)$ and Child A. The survival curves differed significantly among the groups and the curve of Child $\mathrm{B}(9)$ was the worst and tended to go separately from the curve of Child $\mathrm{B}(7-8)$. We then compared $\mathrm{B}(9)$ and $\mathrm{B}(7-8)$ but a statistical difference was not met. The small number of patients in group $\mathrm{B}(9)(\mathrm{n}=11)$ might have contributed to this insignificance despite a trend in the survival curve.

This study did not include patients with de compensated cirrhosis who did not receive TACE as a control group due to the retrospective nature of the study. We used the data from the RCT of Lo et al., which showed 1- and 2-year survival in the TACE group as 57\% and $31 \%$, respectively, which was close to our patient population (59 and $41 \%$ for 1-2-year survival, respectively). In that study, the 1-year survival rate for the non-treatment group was $32 \%$. Therefore, the 1 -year survival rate less than $32 \%$ in Child B (9) was considered not beneficial.

From our study, TACE can be performed in patients with Child B (7) as well as Child B (8). These groups of patients represent patients with early de compensated cirrhosis. However, patients with Child B (9), which represents advanced de compensated cirrhosis, have a poor prognosis after TACE, so TACE is not recommended. This finding corresponds to the current Thai HCC guideline.

To increase patient survival, serum albumin, and tumor size are factors that should be considered in selecting suitable patients for TACE. High serum albumin and tumor size $\leq 5 \mathrm{~cm}$ provide good candidates. TACE with other treatment modalities increases survival. During treatment, patients who can tolerate more than 2 TACE treatments or do not have complications in 4-8weeks after TACE have better survivals. There are some limitations in this study. First, this is a retrospective study. Second, the number of patients with Child B (9) was low and could contribute to the statistical insignificance in survival compared to Child B(7-8). Third, there was no control group (ie, non-treatment group with the same Child score).

\section{Conclusion}

TACE can be performed in HCC patients with early de compensated cirrhosis (Child-Pugh score 7-8) resulting in a 1-year survival close to that of Child A. Regarding conflicting Child-Pugh score recommendation for TACE, our study suggests that TACE can be performed in patients with Child score of up to B8.

\section{Acknowledgements}

We thank the personnel in the Division of Interventional Radiology, Department of Radiology, Faculty of Medicine, Prince of Songkla University for performing TACE in our HCC patients.

\section{Disclosure statements}

We have nothing to disclose.

\section{Conflict of interest}

Author declares that there is no conflict of interest.

\section{References}

1. Heimbach J, Kulik LM, Finn R, et al. Aasld guidelines for the treatment of hepatocellular carcinoma. Hepatology. 2018;67(1):358-380.

2. EASL-EORTC clinical practice guidelines: management of hepatocellular carcinoma. J Hepatol. 2012;56(4):908-943.

3. Omata M, Cheng AL, Kokudo N, et al. Asia-Pacific clinical practice guidelines on the management of hepatocellular carcinoma: a 2017 update. Hepatol Int. 2017;11(4):317-370.

4. Llovet JM, Real MI, Montana X, et al. Arterial embolisation or chemoembolisation versus symptomatic treatment in patients with unresectable hepatocellular carcinoma: a randomised controlled trial. Lancet. 2002;359(9319):1734-1739.

5. Lo CM, Ngan H, Tso WK, et al. Randomized controlled trial of transarterial lipiodol chemo embolization for un resectable hepatocellular carcinoma. Hepatology. 2002;35(5):1164-1171.

6. Llovet JM, Bruix J. Systematic review of randomized trials for un resectable hepatocellular carcinoma: Chemo embolization improves survival. Hepatology. 2003;37(2):429-442.

7. Camma C, Schepis F, Orlando A, et al. Transarterial chemo embolization for un resectable hepatocellular carcinoma: meta-analysis of randomized controlled trials. Radiology. 2002;224(1):47-54.

8. Pawarode A, Tangkijvanich P, Voravud N. Outcomes of primary hepatocellular carcinoma treatment: an 8-year experience with 368 patients in Thailand. J Gastroenterol Hepatol. 2000;15(8):860-864.

9. Sithinamsuwan P, Piratvisuth T, Tanomkiat W, et al. Review of 336 patients with hepatocellular carcinoma at Songklanagarind Hospital. World J Gastroenterol. 2000;6(3):339-343.

10. Somboon K, Siramolpiwat S, Vilaichone RK. Epidemiology and survival of hepatocellular carcinoma in the central region of Thailand. Asian Pac J Cancer Prev. 2014;15(8):3567-3570.

11. Leerapun A, Thaikruea L, Pisespongsa P, et al. Clinical features and prognostic factors for liver cancer from a referral center in northern Thailand. J Med Assoc Thai. 2013;96(5):531-537.

12. Chonprasertsuk S, Vilaichone RK. Epidemiology and treatment of hepatocellular carcinoma in Thailand. Jpn $J$ Clin Oncol. 2017;47(4):294-297.

13. Dufour JF, Bargellini I, De Maria N, et al. Intermediate hepatocellular carcinoma: current treatments and future perspectives. Ann Oncol. 2013;24 Suppl 2:ii24-29.

14. Bolondi L, Burroughs A, Dufour JF, et al. Heterogeneity of patients with intermediate (BCLC B) Hepatocellular Carcinoma: proposal for a subclassification to facilitate treatment decisions. Semin Liver Dis. 2012;32(4):348-359.

15. Kothary N, Weintraub JL, Susman J, et al. Transarterial chemoembolization for primary hepatocellular carcinoma in patients at high risk. J Vasc Interv Radiol. 2007;18(12):1517-1526.

16. Piscaglia F, Terzi E, Cucchetti A, et al. Treatment of hepatocellular carcinoma in Child-Pugh B patients. Dig Liver Dis. 2013;45(10):852-858.

17. Yamakado K, Miyayama S, Hirota S, et al. Sub grouping of intermediatestage (BCLC stage B) hepatocellular carcinoma based on tumor number and size and Child-Pugh grade correlated with prognosis after transarterial chemo embolization. Jpn J Radiol. 2014;32(5):260-265.

18. Cheng AL, Kang YK, Chen Z, et al. Efficacy and safety of sorafenib in patients in the Asia-Pacific region with advanced hepatocellular carcinoma: a phase III randomised, double-blind, placebo-controlled trial. Lancet Oncol. 2009;10(1):25-34. 